\title{
Comparação entre indicadores proxy sintéticos de segurança viária e acidentes de trânsito em interseções semaforizadas de Fortaleza
}

\author{
Janailson Queiroz Sousa ${ }^{1}$, Flávio José Craveiro Cunto ${ }^{2}$ e Marcos William Sasaki ${ }^{3}$
}

\begin{abstract}
Resumo: A simulação de tráfego microscópica tem sido desenvolvida e aplicada nas duas últimas décadas com o foco principal no projeto e operação de sistemas de transporte. Recentemente, devido aos avanços nas técnicas de coleta de dados e algoritmos microscópicos, o potencial da microssimulação como ferramenta para avaliações de segurança tem sido explorado de forma mais extensiva. Este tipo de abordagem pode permitir um melhor conhecimento sobre a cadeia de eventos que precederam a ocorrência dos acidentes, portanto, levando a uma metodologia mais abrangente para estudos de segurança quando comparados aos tradicionais estudos observacionais. Este artigo apresenta um esforço de validação entre colisões traseiras observadas e conflitos de tráfego simulados, refletidos por três medidas de desempenho de segurança (SPM - safety performance measures): tempo para a colisão (TTC - time to collision), taxa de desaceleração para evitar a colisão (DRAC - deceleration rate to avoid the crash) e o índice de potencial para acidentes (CPI crash potencial index). Três interseções semaforizadas e isoladas de vias arteriais da cidade de Fortaleza foram selecionadas para este estudo. Foram coletados três anos de dados de acidentes (2007-2009) para quatro horas do período da manhã (7h00-11h00) e foram comparados com o número de conflitos de tráfego estimado por um experimento de simulação microscópica. Os resultados indicaram que todas as SPM testadas foram capazes de capturar diferenças no número de acidentes entre os três locais de estudo, sugerindo um grande potencial para aplicação dessa abordagem nas análises de segurança. Por outro lado, para alcançar resultados mais amplos e expressivos verificou-se a necessidade de aplicar o método proposto em uma amostra mais representativa. DOI:10.4237/transportes. v20i3.582.
\end{abstract}

Palavras-chave: medidas de desempenho de segurança, segurança viária, simulação microscópica.

\begin{abstract}
Microscopic traffic simulation has been developed and applied over the past two decades with focus towards the design and operation of transportation systems. Recently, due to advancements in data collection techniques and microscopic algorithms, the potential of microscopic simulation as a tool for safety evaluation has been explored more extensively. This type of approach may allow better knowledge regarding the chain of events preceding crash occurrences, therefore leading to a more comprehensive methodology for safety studies when compared to traditional observational studies. This paper presented a validation effort between observed rearend collisions and simulated traffic conflicts as reflected by three SPM namely: Time to Collision - TTC, Deceleration Rate to Avoid the Crash - DRAC and Crash Potential Index - CPI. We collected three years of accident data (2007-2009) for four hours of the morning (7h00-11h00) and were compared with the number of traffic conflicts estimated by a microscopic simulation experiment. The results indicated that all SPM tested were able to capture the differences in the number of accidents among the three different study sites, suggesting a great potential for application of this approach in the safety analyzes. On the other hand, to achieve wider results it's necessary to apply the method proposed in a more representative sample.
\end{abstract}

Keywords: safety performance measures, road safety, microscopic simulation.

\section{INTRODUÇÃO}

Nas duas últimas décadas o uso das técnicas de simulação de tráfego tem sido essencialmente explorado no desenvolvimento de novas tecnologias voltadas para a otimização do desempenho operacional de elementos da infraestrutura dos sistemas de transportes, tais como: interseções semaforizadas, corredores de tráfego e redes urbanas. No campo da segurança viária, o potencial da simulação microscópica na análise de conflitos de tráfego foi inicialmente reconhecido por Darzentas et al. (1980) e ganhou o interesse, principal-

\footnotetext{
1 Janailson Queiroz Sousa, Departamento de Engenharia de Transportes, Universidade Federal do Ceará, Fortaleza, CE, Brasil. (e-mail: janailson@ det.ufc.br).

${ }^{2}$ Flávio José Craveiro Cunto, Departamento de Engenharia de Transportes, Universidade Federal do Ceará, Fortaleza, CE, Brasil. (e-mail: flaviocunto@

det.ufc.br).

${ }^{3}$ Marcos William Sasaki, Departamento de Engenharia de Transportes, Universidade Federal do Ceará, Fortaleza, CE, Brasil. (e-mail: sasaki@
} det.ufc.br).

Manuscrito recebido em 12/5/2012 e aprovado para publicação em 12/11/2012. Este artigo é parte de TRANSPORTES v. 20, n. 3, 2012. ISSN: 2237-1346 (online). DOI:10.4237/transportes.v20i3.582. mente, devido aos recentes desenvolvimentos na modelagem do comportamento humano e na aquisição de dados de tráfego em tempo real.

Em teoria, os modelos microscópicos de tráfego tem o potencial para considerar fatores importantes que influenciam fortemente as ocorrências de acidentes de trânsito, incluindo diferentes aspectos comportamentais dos condutores e as interações individuais entre pares de veículos em tempo real. Isso proporcionaria uma plataforma para o desenvolvimento de estudos de segurança na qual se aplicaria uma abordagem microscópica mais "mecanística" com o objetivo de melhorar o conhecimento da sequência de eventos que antecederam a ocorrência de um acidente. No entanto, alguns aspectos metodológicos desta abordagem precisam ser investigados, tais como:

- Algoritmos microscópicos tradicionais como, modelos de car-following (CFM), aceitação de brecha e mudança de faixa não foram desenvolvidos especificamente para explicar toda a gama de fatores que envolvem a ocorrência de um acidente. Os modelos devem permitir a ocorrência de erros como resultado de falhas na percepção, tomada de decisão e ação dos usuários, causando diferentes níveis de risco nas 
interações entre eles e o ambiente (Archer, 2000; Archer, 2005; Xin et al., 2008);

- A necessidade de ligação objetiva entre o desempenho de segurança simulado e o observado em situações de eminente ocorrência de acidentes de tráfego, com o objetivo de confirmar uma relação válida entre o ambiente simulado e o real aumentando o grau de confiabilidade nos resultados simulados (Cunto e Loureiro, 2011; Saunier et al., 2011).

Com relação ao primeiro aspecto, é possível evidenciar a necessidade de um processo de calibração dos parâmetros dos algoritmos dos simuladores que possa minimizar o fato desses algoritmos não terem sido formatados inicialmente com a capacidade de representar detalhadamente os conflitos de tráfego que antecedem os acidentes viários. Este processo deve buscar adequar o ambiente simulado às características observadas na realidade sob a luz de uma medida de desempenho de segurança observada em campo.

Quanto ao segundo aspecto destaca-se que uma validação do desempenho de segurança simulado além de consolidar este tipo de abordagem também é necessária para verificar que tipos de indicadores proxy podem ser mais bem adequados para refletir o desempenho da segurança, conforme vêm pesquisando Saunier et al. (2011). E isto é um fator relevante tendo em vista que Davis et al. (2008) encontrou indícios de que as ações dos condutores em conflitos que não resultaram em acidentes podem não ser as mesmas em situações que resultaram efetivamente em acidentes.

Medidas de desempenho de segurança (SPM - safety performance measures), também conhecidas como indicadores proxy de segurança ou ainda medidas de segurança substitutas têm sido calculadas a partir de pacotes computacionais de simulação microscópica para refletir eventos de alto risco envolvendo pelo menos um veículo em relação a um ponto projetado de colisão. Dentre as SPM mais documentadas estão o "tempo para colisão" (TTC - time to collision), "taxa de desaceleração para evitar a colisão" (DRAC - Deceleration Rate to Avoid the Crash), "tempo pósinvasão" (PET-post-encroachment time), "índice de potencial para acidentes" (CPI - crash potential index) e outros (Hayward, 1972; Cooper e Ferguson, 1976; Cooper, 1983; Gettman e Head, 2003; Cunto e Saccomanno, 2008). A utilidade da simulação microscópica para avaliar a segurança depende da capacidade destas medidas em capturar as complexas relações comportamentais que podem levar a ocorrência dos acidentes e estabelecer uma ligação entre elas e o potencial risco de acidente observado.

Diante desse contexto, o objetivo principal deste trabalho é apresentar um esforço de validação relativa das SPM, comparando colisões traseiras observadas com três indicadores proxy: TTC, DRAC e CPI para interseções semaforizadas em vias arteriais na cidade de Fortaleza. A hipótese de pesquisa é que se uma SPM simulada reflete o comportamento de alto risco no fluxo de tráfego, então falhas tendem a ocorrer com mais frequência quando os indicadores são consistentemente mais altos. Por outro lado, espera-se que em situações de poucos acidentes estas medidas sejam mais próximas de um valor médio esperado para as condições de tráfego e localização existentes.

\section{MEDIDAS DE DESEMPENHO DA SEGURANÇA VIÁRIA}

O desempenho de segurança simulado é normalmente expresso em termos de indicadores proxy ou medidas de segurança substitutas definidas para refletir eventos de alto risco em relação a um ponto projetado de colisão. Estas medidas são geralmente baseadas em pares de velocidade de veículos e atributos de espaçamento. Uma das principais hipóteses que suportam a utilização das SPM é que se essas medidas forem capazes de detectar situações de alto risco que ocorrem com maior frequência do que acidentes, então resultados de análises do desempenho da segurança viária, estatisticamente confiáveis, seriam possíveis sem a necessidade de dados do histórico de acidentes.

O uso de SPM também constitui, em essência, uma abordagem proativa para estudos de segurança viária, uma vez que é capaz de detectar problemas de segurança antes que resultem em acidentes (Barceló et al., 2003; Dazentas et al., 1980;. Perkins e Harris, 1968). Para este tipo de aplicação três categorias básicas de SPM foram identificadas na literatura da seguinte forma: medidas baseadas no tempo, na energia de frenagem e os índices de segurança (Cunto, 2008).

Medidas com base no tempo são estimadas de acordo com um tempo previsto para uma colisão em potencial de veículos assumindo que os mesmos mantenham as suas velocidades e trajetórias inalteradas. As medidas de tempo mais comuns, com base na literatura são o tempo para a colisão (TTC - time to collision), tempo para o acidente (TTA - time to accident), o tempo de invasão (ET - encroachment time), tempo pós-invasão (PET - post-encroachment time), e tempo de brecha (GT - gap time) (Hayward, 1972; Cooper e Ferguson, 1976; Hydén, 1987; Gettman e Head, 2003). Um problema que limita a aplicação desta medida de segurança é que várias combinações de velocidade e distância podem produzir a mesma medida, portanto, inferências sobre a gravidade dos acidentes são mais problemáticas.

A diferença de velocidade entre os veículos no momento do impacto desempenha um papel importante na gravidade dos acidentes devido à energia cinética do sistema imediatamente antes da colisão. Deste modo, medidas de segurança com base na taxa necessária de redução de velocidade ou energia de frenagem dos veículos têm uma formulação teórica mais adequada para fornecer boas estimativas de potenciais conflitos, além disso, propiciam a produção de uma plataforma de estudos de segurança em que a gravidade é um fator importante. Duas SPM com base na energia de frenagem de veículos em conflito são: taxa de desaceleração para evitar a colisão (DRAC - Deceleration Rate to Avoid the Crash) e a proporção de distância de frenagem (PSD - proportion of stopping distance) (Brian et al., 1978; Archer, 2005; Cooper e Ferguson, 1976; Darzentas et al., 1980; Gettman e Head, 2003).

Desenvolvimentos recentes das técnicas de aquisição de dados em tempo real e o uso crescente da simulação microscópica em estudos de segurança têm estimulado o desenvolvimento de índices de segurança que incorporam uma dimensão temporal às medidas de desempenho tradicionais de segurança. A suposição fundamental subjacente à aplicação desses índices de segurança é que a gravidade dos conflitos e o tempo de exposição correspondente a tal conflito pode proporcionar uma melhor medida de seguran- 
ça que uma única medida, como o menor TTC, o maior DRAC, etc. Alguns dos índices de segurança são: tempo de exposição do tempo para a colisão (TET - time exposed time to collision), tempo integrada do tempo para à colisão (TIT - time integrated time to collision), o parâmetro de densidade de insegurança (UD - the unsafety density parameter) e o índice de potencial para acidentes (CPI - crash potential index) (Barceló et al., 2003; Minderhoud e Bovy, 2001; Cunto, 2008).

Este artigo investiga um indicador de cada categoria de SPM, estimando o número de conflitos longitudinais obtidos a partir de medidas do TTC, DRAC e CPI simulados para três interseções semaforizadas isoladas para pequenos incrementos de tempo $(0,1 \mathrm{~s})$. O TTC foi um dos primeiros indicadores proxy sugeridos, sendo baseados na proximidade temporal entre dois veículos. Por definição o TTC corresponde ao "tempo necessário para dois veículos colidirem se mantidas as suas velocidades e trajetórias" (Hayward, 1972).

Por sua vez, a taxa de desaceleração para evitar a colisão ou DRAC pode ser definida usando relações de tempo/espaço aplicada a um dado par de veículos como a desaceleração exigida pelo veículo seguidor para chegar a uma frenagem necessária ou atingir uma determinada velocidade para evitar uma posterior colisão traseira. De maneira geral, as interações veiculares podem ser classificadas como severas para valores de DRAC acima de $3,0 \mathrm{~m} / \mathrm{s}^{2}$ (McDowell et al. 1983; Hydén, 1996).

Limites para a definição de um conflito de tráfego de traseira foram assumidos de acordo com estudos de Van der Horst (1990), Hyden (1996) e Cunto (2008). Para TTC e DRAC, um conflito de tráfego será atribuído a interações veiculares resultantes em valores de TTC inferiores a $1,5 \mathrm{~s} \mathrm{e}$ DRAC superiores a $3,35 \mathrm{~m} / \mathrm{s}^{2}$. Maiores detalhes sobre a formulação matemática dos indicadores proxy usados neste trabalho são encontrados em Archer (2005), Cunto (2008) e Cunto e Loureiro(2011).
A definição de conflitos usando o índice CPI requer uma suposição sobre a taxa de desaceleração máxima disponível (MADR) para cada veículo e estimativas (ambiente de simulação) do DRAC ao longo do tempo. Para cada veículo na simulação um valor MADR individual é atribuído a partir de uma distribuição normal truncada para carros e caminhões em separado (Tabela 1). Os valores na Tabela 1 foram obtidos a partir de testes de campo para veículos diferentes, com velocidades iniciais de 80 a $100 \mathrm{~km} / \mathrm{h}$ chegando à parada completa (Neilsen, J., 2007; MOVIT, 2007). Os valores de DRAC, por outro lado, foram obtidos a partir de interações traseiras de veículos como representado pela combinação de atributos geométricos e de tráfego e as relações com os algoritmos microscópicos de car-following, aceitação de brecha e mudança de faixa. Neste caso, um conflito de tráfego é detectado quando um determinado veículo tem um esforço de frenagem necessário para evitar a colisão maior do que sua capacidade máxima de frenagem, ou seja, DRAC> MADR.

Tabela 1. Parâmetros assumindo MADR - Distribuição normal truncada

\begin{tabular}{lrc}
\hline Parâmetros da distribuição & Carros & $\begin{array}{l}\text { Onibus } \boldsymbol{e} \\
\text { Caminhões }\end{array}$ \\
MADR & 8,45 & 5,01 \\
Média $\left(\mathrm{m} / \mathrm{sec}^{2}\right)$ & 1,40 & 1,40 \\
Desvio Padrão $\left(\mathrm{m} / \mathrm{sec}^{2}\right)$ & 13,45 & 7,98 \\
Limite superior $\left(\mathrm{m} / \mathrm{sec}^{2}\right)$ & 3,45 & 2,05 \\
Limite inferior $\left(\mathrm{m} / \mathrm{sec}^{2}\right)$ & 100 & 100 \\
Distância Máx. de Conflito (m) & \\
\hline
\end{tabular}

\section{METODOLOGIA PROPOSTA PARA VALIDAÇÃO RELATIVA SPM/ACIDENTES}

A metodologia proposta para os testes de exploração da validação relativa entre acidentes observados e o desempenho de segurança simulado é sintetizada pelo fluxograma ilus-

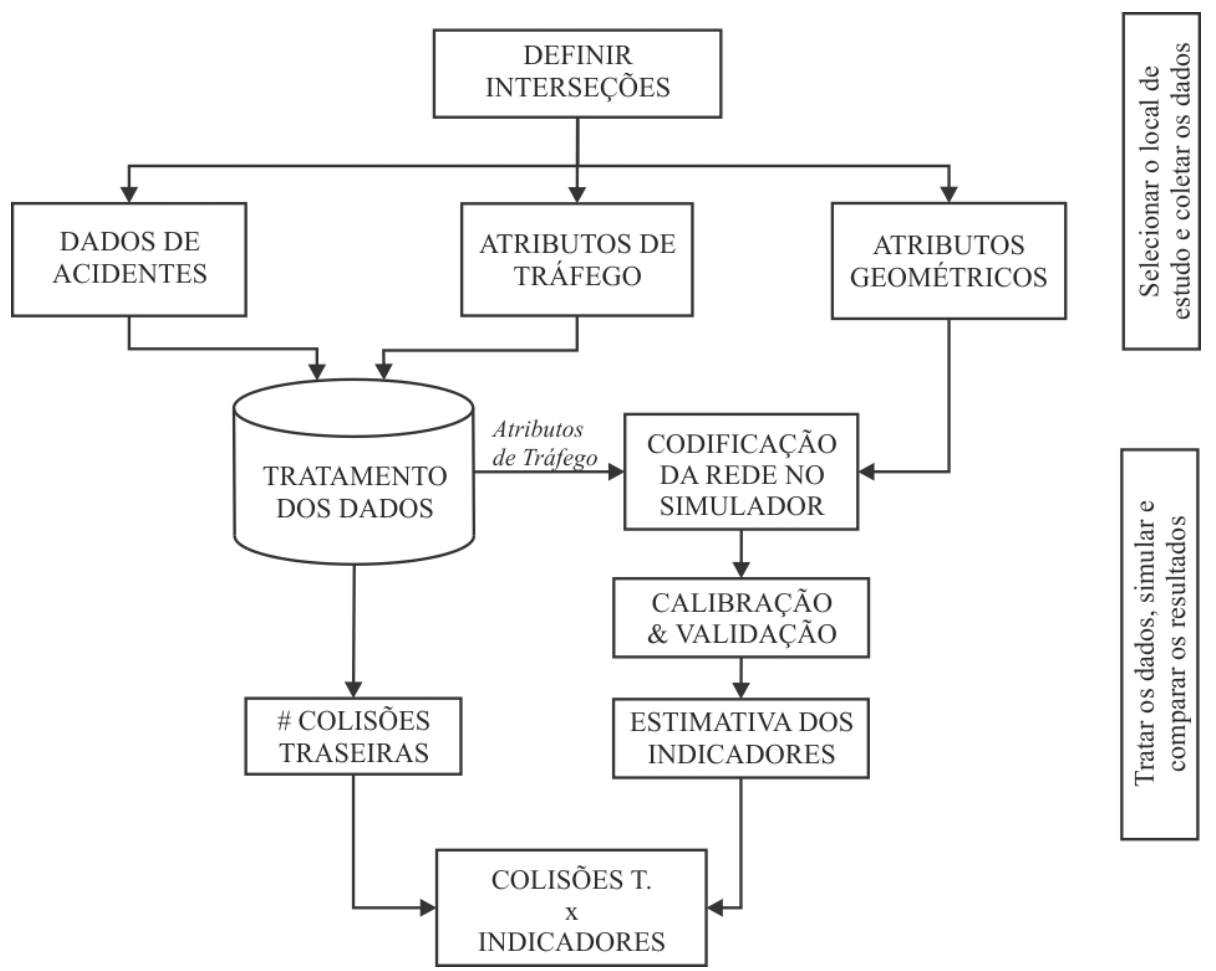

Figura 1. Fluxograma do processo de validação relativa das SPM 
trado na Figura 1. Este fluxograma é composto por sete etapas: 1) Definição de locais (interseções) e respectiva área de interesse a ser investigada, 2) Obtenção de dados sobre os acidentes para áreas de estudo, 3) Coleta de dados dos atributos geométricos e de tráfego, 4) Codificação de rede microscópica, 5) Calibração e validação de algoritmos microscópicos, 6) Estimativa das SPM para as interseções selecionadas, e 7) Comparação entre os dados de acidentes e dos conflitos simulados.

\subsection{Definição dos locais e áreas de interesse}

Para testar a validade relativa entre os indicadores proxy e os dados de acidentes, três interseções foram selecionadas com base no tipo de controle de tráfego, condições de estacionamento e influência de outros cruzamentos. As interseções selecionadas são controladas por semáforos de dois e três estágios que ordenam os fluxos das vias alternando a preferência de passagem entre elas. Partindo da premissa de que não há a problemas com o avanço semafórico, todas as colisões observadas nessas interseções tiveram como fator preponderante as paradas ocasionadas pela alternância do direito de passagem.

A influência das condições de estacionamento nas colisões traseiras foi considerada uma fonte potencial de viés desses acidentes. Os atuais algoritmos microscópicos não consideram explicitamente estas manobras em sua formulação, portanto, um importante critério para selecionar cruzamentos candidatos foi a não ocorrência de estacionamentos dentro a área de interesse em cada aproximação.

O terceiro critério de seleção foi relacionado a dependência espacial das interseções. Neste caso, foram selecionadas interseções com uma distância considerável de outros cruzamentos sinalizados ( $>200 \mathrm{~m})$ para evitar o agrupamento de veículos da interseção a jusante e a introdução de uma dependência espacial entre as entidades. Esses fatores produziriam um nível extra de esforço para serem adequadamente considerados pelos algoritmos atuais de microssimulação de tráfego.

A Figura 2 ilustra as interseções selecionadas, a saber: Int. 168 (av. Dedé Brasil x av. dos Expedicionários), Int. 243 (av. Murilo Borges x av. Rogaciano Leite) e Int. 250 (av. Murilo Borges x av. Raul Barbosa). Todas as interseções são formadas por vias arteriais com canteiro central e as aproximações variam de duas a três faixas de tráfego por pista de rolamento.

As áreas de interesse foram definidas para cada cruzamento para abranger o comprimento médio da fila medido, a partir da linha de retenção, para cada aproximação durante o período da manhã $(07 \mathrm{~h} 00$ - 11h00). O comprimento da fila média foi obtido a partir do histórico do banco de dados da divisão de controle de tráfego em área de Fortaleza (CTAFOR) e por medições em campo, os valores variaram entre 80 e 200 metros.

\subsection{Banco de dados de acidentes para as áreas de interesse}

Neste estudo, três anos de dados de acidentes (2007-2009) foram coletados a partir do sistema municipal de informações acidentes de trânsito (SIAT-FOR) para serem comparados com as SPM obtidas com a microssimulação. Inicialmente, relatórios completos de acidentes foram extraídos independentemente da definição do tipo de acidente, dia da semana e os veículos envolvidos. Uma série de filtros foi então aplicada para considerar apenas as colisões traseiras que ocorreram nos horários e condições adequadas para as análises (Tratamento dos Dados).

A fim de minimizar as flutuações aleatórias devido ao comportamento diferenciado de condução nos fins de semana, acidentes registrados nos sábados e domingos foram removidos do conjunto de dados. Um aspecto importante observado em muitas cidades brasileiras é o uso considerável de motocicleta para fins comerciais e das bicicletas como meio de transporte de operários. Isso introduz um número significativo de colisões envolvendo os usuários que não podem ser adequadamente representados em algoritmos de tráfego microscópicos, por este motivo os acidentes envolvendo as motocicletas e as bicicletas também foram removidos do conjunto de dados. A referida dificuldade também influenciou a não consideração dos acidentes do tipo atropelamento nesta análise. Além disso, o fato das interseções analisadas serem semaforizadas garante uma maior segurança na travessia dos pedestres pela alternância no controle do direito de passagem de cada aproximação. Por fim, uma análise prévia também apontou que a maioria das ocorrências de acidentes do tipo atropelamento foi registrada fora do horário considerado para esta análise (07h00 11h00).

Por fim, para coleta dos acidentes dentro das áreas de interesse foram considerados dois tipos de localização, como segue: 1) Os acidentes dentro da área da interseção e 2) Os acidentes dentro da área abrangida pelo comprimento médio da fila (segmentos). Para identificar a localização real de acidentes observados em segmentos, dois tipos de relatórios foram solicitados: o relatório com base no número da
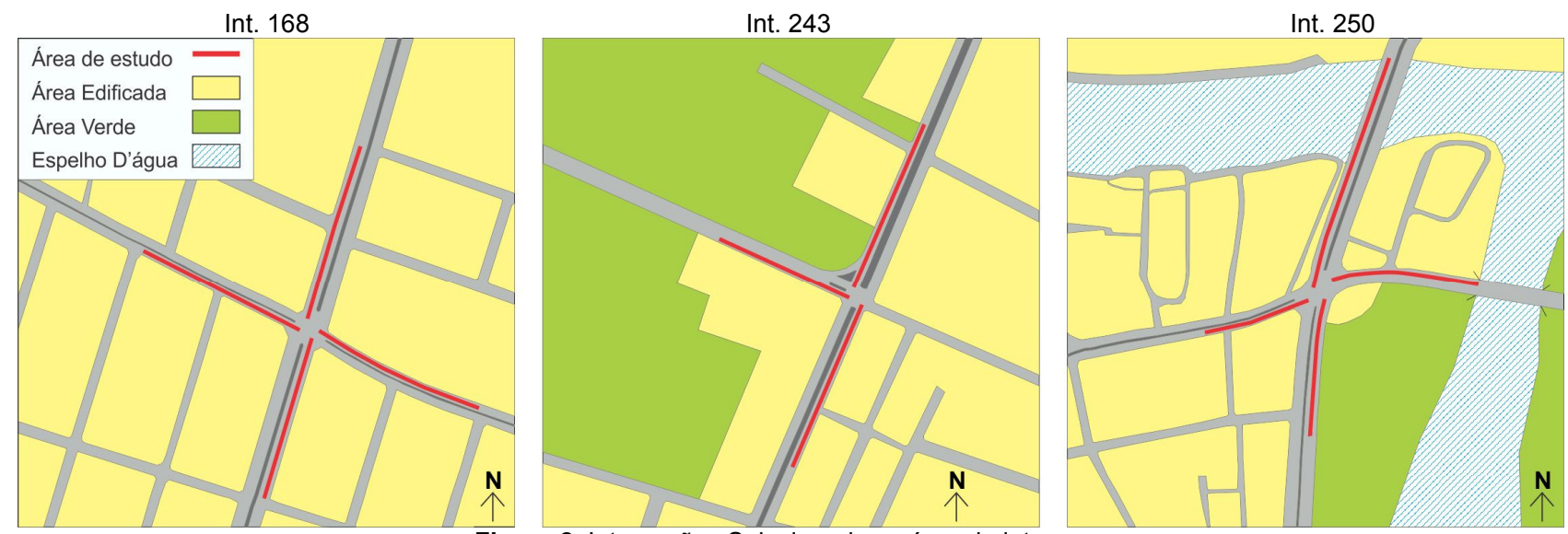

Figura 2. Interseções Selecionadas e área de interesse 
Tabela 2. Total de colisões registradas e "válidas" (2007-2009)

\begin{tabular}{|c|c|c|}
\hline & $\begin{array}{l}\text { \# TOTAL DE } \\
\text { COLISÕES }\end{array}$ & $\begin{array}{l}\text { \# COLISÕES } \\
\text { TRASEIRAS }\end{array}$ \\
\hline INT \#168Manhã & 127 & 46 \\
\hline INT \#243Manhã & 34 & 14 \\
\hline INT \#250Manhã & 112 & 56 \\
\hline
\end{tabular}

residência ou comércio e o relatório com base nos pontos de referência fornecidos pelo sistema SIAT-FOR. A Tabela 2 apresenta o resumo das colisões registradas, bem como os valores considerados para os três anos da análise.

\subsection{Atributos geométricos e de tráfego}

Os atributos geométricos para a codificação da rede foram obtidos utilizando o software Google Earth ${ }^{\circledR}$ sendo complementados por medições em campo para confirmar os aspectos geométricos, tais como o número e a largura das faixas, largura dos separadores centrais e os ângulos de intersecção. Os dados de tráfego foram obtidos a partir do banco de dados do sistema adaptativo de controle de tráfego (ATCS) SCOOT/ASTRID da divisão de controle de tráfego em área de Fortaleza (CTAFOR) sendo ainda complementados por inspeções em campo. Dentre os dados coletados incluem-se o fluxo de tráfego médio agregado em amostras de 15 minutos por aproximação, duração do ciclo, atraso médio etc. A Tabela 3 apresenta uma amostra dos atributos de tráfego obtidos junto ao CTAFOR para a interseção \# 168. Na primeira coluna das tabelas 3 e 4 os algarismos representam o número da interseção e as letras a, b, c e d representam respectivamente as a aproximações norte, leste, sul e oeste.

O conjunto de dados utilizados para estimar atributos do tráfego foi obtido a partir de uma amostra de 80 dias típicos do ano de 2009. Semelhante ao conjunto de dados de colisão a amostra dos dados de tráfego foi filtrada e compilada para os dias e horas de pesquisa de interesse (Tratamento dos Dados). Além disso, o conjunto de dados foi estatisticamente tratado para identificar outliers e observações em falta utilizando a ferramenta de box-plot como proposto por Oliveira (2004). Para complementar as informações básicas fornecidas pelo ATCS foram usadas câmeras de TV do circuito interno do CTAFOR e ainda inspeções em campo no período de interesse (7h00-11h00) para cada cruzamento com o objetivo de estimar a percentagem de veículos pesados (caminhões e ônibus), bem como classificação direcional do tráfego. A seguir na Tabela 4 são apresentados os dados básicos do tráfego coletados para os locais de estudo de cada aproximação.

\subsection{Plataforma para estimativa das SPM}

A plataforma de simulação para estimar as medidas de segurança de desempenho utilizados nesta pesquisa foi VISSIM 5.30-03 C. A plataforma de simulação VISSIM é baseada em algoritmos psicofísicos de condução cujo modelo de car-following considera quatro tipos de regimes onde os condutores podem ajustar seu espaçamento e velocidade desejada através de mudanças em suas taxas de aceleração e desaceleração. Foi reconhecido que, apesar de suas limitações para refletir acidentes de forma direta, estes algoritmos são capazes de gerar uma grande variedade de interações veiculares refletindo uma heterogeneidade considerável no fluxo de tráfego que permitem as análises de segurança viária (Gettman e Head, 2003; Archer, 2005; Cunto, 2008).

Atributos geométricos e de tráfego foram codificados no VISSIM para todos os cruzamentos durante as quatro horas de investigação. O fluxo de tráfego médio para cada aproximação foi considerado em intervalos de 15 minutos (oito intervalos para cada período) para explicar a variabilidade de tráfego usando as informações descritas na seção anterior.

\subsection{Calibração/Validação}

Cunto e Saccomanno (2008) apresentaram um esforço para calibrar e validar os parâmetros do VISSIM dos modelos de car-following, aceitação de brecha, e mudança de faixa com base em observações do CPI obtidos a partir de dados de

Tabela 3. Amostra de atributos de tráfego para interseção \# 168

\begin{tabular}{lllllll}
\hline & Dia e Data & $\begin{array}{l}\text { Tempo } \\
\text { Inicial } \\
\text { (hh:mm) }\end{array}$ & $\begin{array}{l}\text { Tempo } \\
\text { Final } \\
\text { (hh:mm) }\end{array}$ & $\begin{array}{l}\text { Fluxo } \\
\text { Médio } \\
\text { (veh/h) }\end{array}$ & $\begin{array}{l}\text { Atraso } \\
\text { Médio } \\
\text { (veh) }\end{array}$ & $\begin{array}{l}\text { Atraso } \\
\text { Médio } \\
\text { (s) }\end{array}$ \\
\hline 00168:aximação & FR 6-Mar-2009 & $07: 00$ & $07: 15$ & 688 & 21,9 & 114,5 \\
00168:a & MO 9-Mar-2009 & $07: 00$ & $07: 15$ & 735 & 21,9 & 107,2 \\
00168:a & TU 10-Mar-2009 & $07: 00$ & $07: 15$ & 762 & 20,5 & 96,8 \\
00168:a & WE 11-Mar-2009 & $07: 00$ & $07: 15$ & 785 & 20,9 & 95,8 \\
\hline
\end{tabular}

Tabela 4. Resumo do Tráfico atributos de entrada

\begin{tabular}{|c|c|c|c|c|c|c|}
\hline \multirow[b]{2}{*}{ Aproximação } & \multirow[b]{2}{*}{$\begin{array}{l}\text { Fluxo Médio } \\
\text { (veh/h) }\end{array}$} & \multicolumn{3}{|c|}{ \% Conversão } & \multirow[b]{2}{*}{$\begin{array}{l}\text { \% Caminhões } \\
\text { e Ônibus }\end{array}$} & \multirow[b]{2}{*}{$\begin{array}{l}\text { Tempo de } \\
\text { Ciclo (s) }\end{array}$} \\
\hline & & Esq. & $\begin{array}{l}\text { Fren- } \\
\text { te }\end{array}$ & Dir. & & \\
\hline 00168:a & 811 & 21 & 72 & 7 & 6 & 176 \\
\hline 00168:b & 793 & - & 82 & 18 & 14 & 176 \\
\hline 00168:c & 1309 & 20 & 75 & 5 & 8 & 176 \\
\hline 00168:d & 733 & - & 86 & 14 & 16 & 176 \\
\hline 00243:a & 1095 & 27 & 30 & 43 & 2 & 160 \\
\hline 00243:c & 874 & 34 & 64 & 2 & 6 & 160 \\
\hline 00243:d & 705 & 35 & 59 & 6 & 4 & 160 \\
\hline 00250:a & 1610 & - & 86 & 14 & 6 & 176 \\
\hline $00250: \mathrm{b}$ & 910 & 35 & 53 & 12 & 4 & 176 \\
\hline 00250:c & 1017 & - & 78 & 22 & 10 & 176 \\
\hline 00250:d & 768 & 35 & 64 & 1 & 7 & 176 \\
\hline
\end{tabular}


Tabela 5. Calibração dos parâmetros de entrada do VISSIM (Cunto, 2008)

\begin{tabular}{lll}
\hline Parâmetro de entrada & Valor Calibrado & Descrição \\
\hline Desired deceleration & $-2,6$ & $\begin{array}{l}\text { Máxima desaceleração }\left(\mathrm{m} / \mathrm{s}^{2}\right) \text { que os condutores estão dispostos } \\
\text { a aplicar em condições normais (sem emergência). }\end{array}$ \\
\hline $\mathrm{CC} 0$ & 3,0 & $\begin{array}{l}\text { Distancia de parada (m); define a distância desejada entre os } \\
\text { carros parados. }\end{array}$ \\
\hline & & $\begin{array}{l}\text { Tempo de Headway (s); definido como o tempo mínimo que o } \\
\text { condutor deseja manter do veículo líder; quanto maior o valor } \\
\text { mais cauteloso é o condutor; CC0 e CC1 são combinados para } \\
\text { expressar a distância de segurança. }\end{array}$ \\
\hline
\end{tabular}

rastreamento veicular. Os resultados deste exercício de calibração/validação permitiram uma melhor estimativa para os parâmetros que foram identificados como estatisticamente significativos para explicar o desempenho da segurança viária obtida a partir da microssimulação. Estes valores foram assumidos para esta pesquisa e encontram-se resumidos na Tabela 5. Nesse trabalho considerou-se que os parâmetros calibrados podem ser utilizados para TTC e DRAC uma vez que os dois indicadores utilizam em sua formulação o mesmo tipo de informação (headways e velocidades) e, além disso, o indicador CPI utiliza o indicador DRAC em sua formulação. Valores padrões do VISSIM foram usados para os parâmetros de entrada que não foram considerados como estatisticamente significativos para a simulação do desempenho da segurança viária.

\subsection{Estimativa das SPM}

Para cada cenário de simulação (01 para cada interseção) 10 simulações foram realizadas utilizando números sementes diferentes para estimar a variabilidade entre as medidas de TTC, DRAC e CPI. Informações de cada veículo como coordenadas do veículo, tipo de veículo, velocidade, distância, aceleração / desaceleração taxa, headway, veículo da frente, o veículo anterior e outros foram registrados a cada $0,1 \mathrm{~s}$ (intervalo de tempo) para estimar as SPMs. Estas variáveis individuais dos veículos foram compiladas e processadas através de um aplicativo codificado em visual basic.net denominado de EIVISSIM para obter as estimativas de TTC, DRAC e CPI para cada 0,1 segundos, bem como os conflitos de tráfego derivados de cada uma, conforme descrito anteriormente.

\section{ANÁLISE DOS RESULTADOS}

Para o exercício de validação, o número médio de conflitos longitudinais resultantes da experiência de simulação (4 horas de simulação para cada uma das 10 sementes diferentes) foi comparado com o número de acidentes ocorridos nas mesmas horas de interesse durante três anos (2007-2009) para cada interseção. Para efetuar a comparação, o número médio de conflitos foi expandido considerando o número de dias da semana, exceto feriados, dos três anos de referência. Assim, o valor do indicador final conflitos obtidos para cada período de análise foi ampliado por um valor constante de 739, que representa a soma do número de dias dos três anos de referência excluindo finais de semana e feriados. A Tabela 6 apresenta o número de acidentes observados e número médio de conflitos estimados para o período de três anos. A tabela apresenta ainda os coeficientes de variação para cada SPM.

A análise comparativa entre cruzamentos sugere uma consistência bastante razoável entre o número estimado de conflitos e número observado de acidentes. Em geral, níveis mais altos de acidentes observados implicaram em maiores números de conflitos de tráfego para todas as SPM testadas. Tendência semelhante foi observada com relação ao fluxo médio, acidentes e valores das SPM, reforçando a noção geralmente aceita de que os acidentes e conflitos de tráfego tendem a aumentar com a exposição.

Os resultados da Tabela 6 também apontam que Interseção \#243 apresentou o menor número de conflitos por veículo, independentemente do indicador de segurança, quando comparada com as outras interseções. Este fato foi investigado através de um teste de comparação de médias que indicou ser possível refutar a hipótese nula de que a média dos conflitos da interseção \#243 é igual à média das demais interseções. Isto pode ser explicado por dois fatores, a saber: 1) A existência de apenas três aproximações na intersecção, resultando em menos interações na área de estudo, e 2) Um movimento de conversão à direita livre na aproximação norte, portanto, resultando em um menor número de conflitos longitudinais na faixa da direita dessa aproximação.

Complementando as informações apresentadas na Tabela 6, na Figura 3 é apresentada uma comparação gráfica entre os acidentes e os indicadores obtidos. Esta comparação sugere que quando se confrontam os resultados das três SPM com o número de acidentes válidos das interseções, observa-se que os limites do TTC e do DRAC $\left(1,5 \mathrm{~s}\right.$ e $3,35 \mathrm{~m} / \mathrm{s}^{2}$, respectivamente) produziram consideravelmente mais veículos em conflitos em relação ao CPI. Além disso, pela Tabela 6 , também é possível observar que os conflitos estimados pelo CPI apresentaram a maior variabilidade entre os indicadores. Esta variabilidade pode ser atribuída ao fato deste indicador possuir dois componentes de aleatoriedade, sendo o primeiro associado ao processo de geração de veículos na simulação (geração de semente aleatória) e o segundo associado a distribuição normal truncada apresenta-

Tabela 6. Colisões observadas e conflitos simulados

\begin{tabular}{|c|c|c|c|c|c|c|c|c|c|}
\hline \multirow{2}{*}{$\begin{array}{l}\text { Interse- } \\
c ̧ \tilde{a} o\end{array}$} & \multirow{2}{*}{$\begin{array}{l}\text { \# Colisões } \\
\text { Traseiras }\end{array}$} & \multirow{2}{*}{$\begin{array}{l}\text { Fluxo Médio } \\
\text { (veh/h) }\end{array}$} & \multirow{2}{*}{$\begin{array}{l}\text { \# Veículos } \\
\text { gerados }\end{array}$} & \multicolumn{3}{|c|}{ \# Conflitos x $10^{3}$ (média) } & \multicolumn{3}{|c|}{ Coeficiente de variação } \\
\hline & & & & TTC & $D R A C$ & $C P I$ & $T T C$ & $D R A C$ & $C P I$ \\
\hline INT\#168 & 46 & 3577 & 14327 & 287,2 & 51,1 & 6,1 & 0,04 & 0,13 & 0,38 \\
\hline INT\#243 & 14 & 2563 & 9763 & 162,1 & 33 & 2,6 & 0,07 & 0,12 & 0,70 \\
\hline INT\#250 & 56 & 4283 & 16550 & 330,0 & 89,2 & 13,2 & 0,04 & 0,06 & 0,22 \\
\hline
\end{tabular}



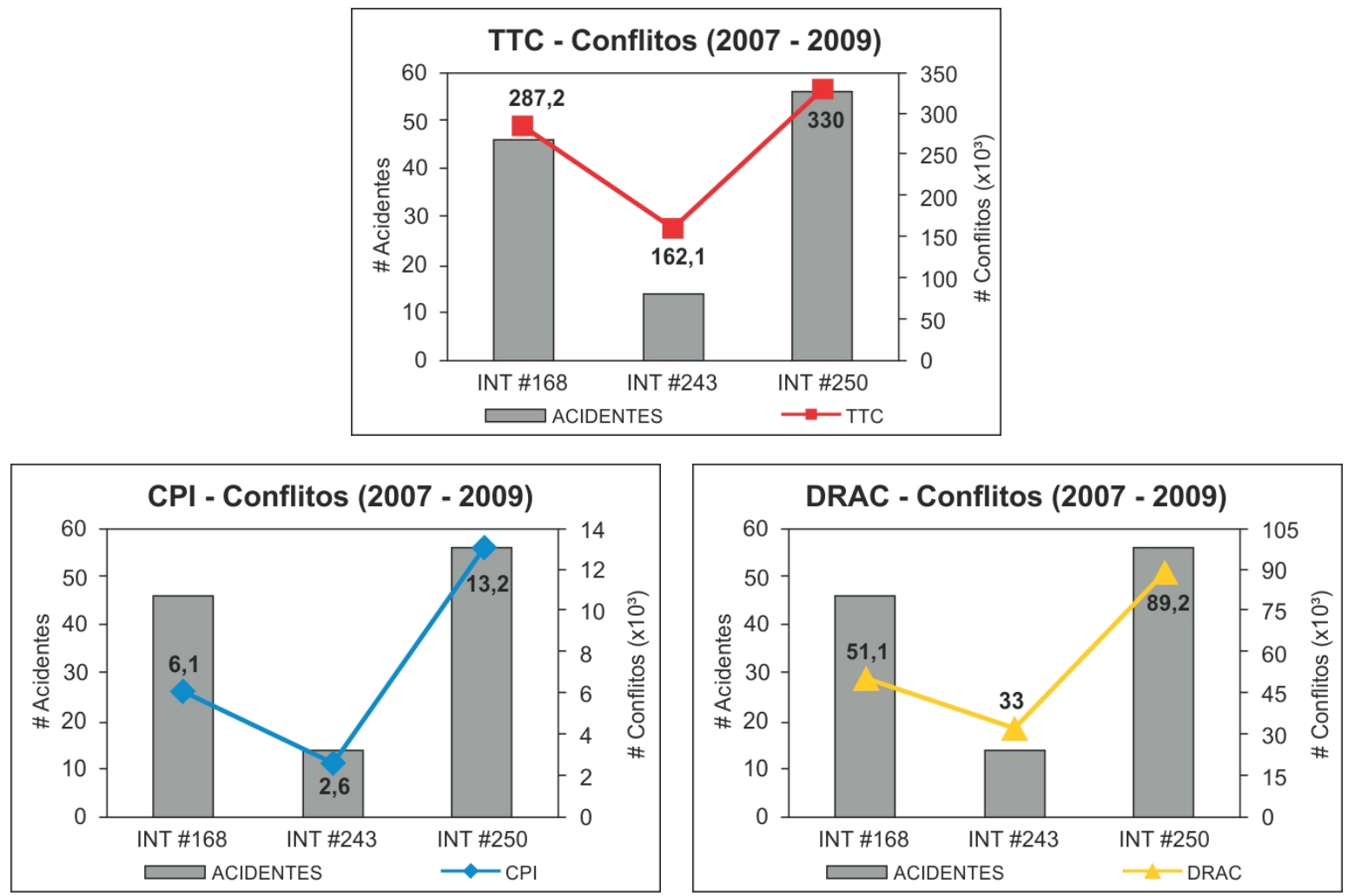

Figura 3. Números de conflitos por interseção

da na Tabela 1 .

As diferenças entre o número médio de conflitos e sua variabilidade (desvio padrão) em função do indicador mostram indícios de que o número mínimo de simulações necessárias para detectar (estatisticamente) diferenças no desempenho da segurança viária pode variar consideravelmente entre os indicadores TTC, DRAC e CPI. Desta forma, um experimento complementar foi planejado para cada uma das interseções do presente estudo no intuito de investigar a sensibilidade dos indicadores em termos no número de simulações necessárias para confirmar alterações de 5\%, $10 \%$ e $20 \%$ no valor médio de conflitos. Para realizar essa verificação foram utilizadas as diretrizes do Manual da FHWA (2004) para aplicação de modelagem microscópica do tráfego. Segundo o Manual, para estimar o número mínimo de simulações necessárias para realizar um experimento são necessárias três informações básicas: 1. Desvio padrão da medida de desempenho procurada na amostra; 2. O comprimento do intervalo de confiança e 3 . O nível de confiança.

O número de simulações necessárias para estimar uma medida de desempenho deve ser realizado por um processo interativo em que deve ser calculado o desvio padrão de uma amostra inicial dessa medida (no mínimo seis simulações), em seguida deve ser determinado o nível de confiança (normalmente 95\%) e se estabelecer a amplitude de um intervalo de confiança que deve ser definido pelo analista de acordo com objetivo do experimento.

Definidas as informações preliminares aplica-se a Equação (1) a seguir para determinar o número mínimo de simulações:

$$
C I_{i-\alpha \%}=2 * t_{(1-\alpha / 2), N-1} \frac{s}{\sqrt{\mathrm{N}}}
$$

em que,

$C I_{(1-\alpha) \%}:(1-\alpha) \%$ intervalo de confiança para a média verdadeira, onde alfa é igual a probabilidade do intervalo de confiança conter o valor verdadeiro;

$t_{(1-\alpha / 2), N-l}$ : estatística t de Student para a probabilidade da soma dos erros de dois lados da curva para alfa com N-1 graus de liberdade, onde N é igual ao número de repetições; e

$S$ : desvio padrão da amostra.

Com base na amostra inicial das dez simulações efetuadas estimou-se o número mínimo de simulações necessário para inferir os resultados das SPM testadas neste trabalho (TTC, DRAC e CPI) para um nível de confiança de 95\% e um intervalo de confiança de 5, 10 e 20\% (Tabela 7).

Os resultados apresentados na Tabela 7, considerando o número de simulações realizadas neste trabalho (10), indicam que para um intervalo de confiança de $5 \%$ não é possível estabelecer inferências confiáveis para nenhuma das

Tabela 7. Número mínimo de simulações para confirmar diferenças no DSV

\begin{tabular}{|c|c|c|c|c|c|c|c|c|c|c|c|c|}
\hline \multirow[t]{2}{*}{$\# I N T$} & \multicolumn{3}{|c|}{ MÉDIA (DESVIO PADR ÃO) } & \multicolumn{3}{|c|}{ 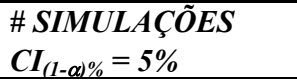 } & \multicolumn{3}{|c|}{ 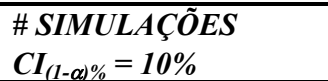 } & \multicolumn{3}{|c|}{ 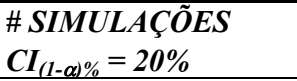 } \\
\hline & TTC & $D R A C$ & $C P I$ & TTC & $D R A C$ & $C P I$ & TTC & $D R A C$ & $C P I$ & TTC & $D R A C$ & $C P I$ \\
\hline INT \#168 & $388,6(15,9)$ & $69,1(9,3)$ & $8,2(3,1)$ & 17 & 148 & 1150 & 6 & 39 & 280 & 3 & 12 & 74 \\
\hline INT \#243 & $219,4(15,3)$ & $44,6(5,3)$ & $3,5(2,5)$ & 42 & 118 & 3900 & 13 & 32 & 1000 & 5 & 10 & 251 \\
\hline INT\#250 & $446,6(18,6)$ & $120,7(7,5)$ & $17,8(3,9)$ & 16 & 35 & 390 & 7 & 11 & 100 & 4 & 5 & 28 \\
\hline
\end{tabular}


três SPM testadas, sendo, portanto necessárias um número maior de simulações para este intervalo. Já para o segundo intervalo de confiança (10\%) os resultados indicam que já são possíveis inferências confiáveis para o indicador TTC, entretanto ainda sendo necessário um número maior de simulações para os demais indicadores.

O terceiro intervalo de confiança $(20 \%)$ foi o que "acomodou", de maneira geral, os indicadores TTC e DRAC obtidos com apenas 10 simulações. Em outras palavras, pode-se afirmar que cenários testados com apenas 10 simulações devem ter valores médios de conflitos com diferença superior a $20 \%$ para serem detectados estatisticamente. A tabela 7, ainda, indica que inferências confiáveis sobre o desempenho da segurança viária com o indicador proxy CPI, para as três interseções analisadas, só são possíveis com um grande número de simulações, chegando a ordem de 4000 simulações para a interseção 243 com um intervalo de confiança de $5 \%$. Esta constatação era esperada tendo em vista a alta variabilidade observada nos resultados deste indicador, conforme apresentados na Tabela 6. Desta forma, os resultados apresentados, sugerem que o CPI é o indicador menos suscetível a detectar pequenas diferenças no DSV quando utilizado na comparação de diferentes alternativas/cenários em se tratando de interseções.

\section{CONSIDERAÇÕES FINAIS}

Este artigo apresentou um esforço de validação entre colisões traseiras observadas e conflitos de tráfego simulados como refletido por três SPM, a saber: TTC, DRAC e CPI. A metodologia proposta foi aplicada em três interseções isoladas e semaforizadas da cidade de Fortaleza, Brasil. Três anos de dados de acidentes (2007-2009) para quatro horas do período da manhã (7h00 - 11h00) foram comparados com a estimativa do número de conflitos de tráfego obtido para as mesmas condições por um experimento de simulação microscópica.

Os resultados globais dessa pesquisa indicaram um relativo nível de consistência entre o número estimado de conflitos e o número observado de colisões traseiras. Em geral, níveis mais altos de acidentes observados implicaram em maiores número de conflitos de tráfego para todas as três SPM testadas. Todos os três indicadores obtidos sinteticamente reproduziram o mesmo ranking relativo das interseções testadas ao se utilizar com medida de desempenho a frequência de acidentes de trânsito.

Ressalta-se que, em virtude da grande variabilidade inerente aos acidentes de trânsito, resultados mais consistentes sob a forma de modelos estatísticos relacionando características das vias e o número de conflitos com os acidentes de trânsito, necessitam de uma amostra de interseções mais significativa. Recomenda-se, desta forma, a ampliação do número de interseções e do período de análise para obtenção de resultados mais robustos.

Uma importante análise realizada neste trabalho discorreu sobre o número de simulações necessárias para inferir com relativa confiança sobre o desempenho da segurança viária (DSV) obtido com o uso das SPM. Esta análise demonstrou que os resultados obtidos neste experimento para os indicadores TTC e DRAC devem ser apontados como representativos sob um intervalo de confiança de $20 \%$. Outro fator importante nesta análise foi o comportamento do índice CPI, o qual, por seu baixo valor médio de conflitos e elevada variabilidade normalmente vai requerer um elevado número de simulações para se garantirem inferências confiáveis para o DSV no ambiente pesquisado (interseções). Acredita-se que este comportamento pode ser atribuído ao fato do indicador CPI considerar em seu cálculo dois distintos componentes aleatórios (DRAC e MADR).

A análise dos microssimuladores e o desenho do experimento revelaram ainda aspectos operacionais e comportamentais do ambiente urbano nacional como a representação das manobras de estacionamento e o uso da motocicleta os quais carecem de um esforço de modelagem mais aprofundado. Acredita-se que os resultados apresentados aliados ao aperfeiçoamento das técnicas de coleta de dados veiculares e ao aumento da capacidade computacional, tornarão a utilização de plataformas de microssimulação na modelagem do desempenho da segurança viária cada vez frequente no cenário Brasileiro.

\section{REFERÊNCIAS BIBLIOGRÁFICAS}

Archer, J. (2000) Developing the potential of micro-simulation modelling for traffic safety assessment. In Proceedings of the $13^{\text {th }}$ ICTCT Workshop.

Archer, J. (2005) Indicators for traffic safety assessment and prediction and their application in micro-simulation modelling: A study of urban and suburban intersections. Tese (Doutorado). Department of Infrastructure, Royal Institute of Technology. Stockholm.

Barceló, J., A. Dumont, L. Montero, J. Perarnau e A. Torday (2003) Safety indicators for microsimulation-based assessments. In $82^{\text {nd }}$ Annual Meeting of the Transportation Research Board.

Brian L., B. Allen, T. Shin e P. J. Cooper (1978) Analysis of traffic conflicts and collisions. Transportation Research Record: Journal of the Transportation Research Board, n. 667, p. 67-74.

Cooper, D. F. e N. Ferguson (1976) Traffic studies at t-junctions - a conflict simulation model. Traffic Engineering and Control, v. 17, p. 306-309.

Cooper, P. J. (1983) Experience with traffic conflicts in Canada with emphasis on post encroachment time techniques. In Proceedings of the NATO Adavanced Research Workshop on International Calibration Study of Traffic Conflict Technique.

Cunto, F. J. C. e C. F. G Loureiro (2011) O uso da microssimulação na avaliação do desempenho da segurança viária. Transportes, v. 19, n. 3, p. 5-11

Cunto, F. J. C. e F. F. Saccomanno (2008) Calibration and validation of simulated vehicle safety performance at signalized intersections. Accident Analysis and Prevention, v. 40, p. 1171-1179. DOI: $\underline{10.1016 / \text { j.aap.2008.01.003. }}$

Cunto, F. J. C. (2008) Assessing Safety Performance of Transportation Systems using Microscopic Simulation. Tese (Doutorado). Department of Civil and Environmental Engineering, University of Waterloo, Ontário.

Darzentas, J., D. Cooper, P. Storr e M. McDowell (1980) Simulation of road traffic conflicts at t-junctions. Simulation, v. 34, p. 155-164. DOI: $10.1177 / 003754978003400505$.

Davis, G. A., J. Hourdos, e H. Xiong. (2008) Outline of Causal Theory of Traffic Conflicts and Collisions. Presented at 87th Annual Meeting of the Transportation Research Board, Washington, D.C.

FHWA (2004) Guidelines for Applying Traffic Microsimulation Modeling Software, Final Report, Publication No FHWA-HRT-04-040, Federal Highway Administration - FHWA, USA.

Gettman, D. e L. Head (2003) Surrogate safety measures from traffic simulation models. Technical report, Publication No FHWA-RD-03050Federal Highway Administration - FHWA. USA.

Hayward, J. C. (1972) Near-miss determination through use of a scale of dange. Highway Research Record, v. 384, p. 24-34.

Hydén, C. (1987) The development of a method for traffic safety evaluation: The swedish traffic conflicts technique. Bulletin 70. Department of Traffic Planning and Engineering, Lund University, Lund, Sweden.

Hydén, C. (1996) Traffic safety work with video-processing. Technical report. Transportation Department, University Kaiserslautern.

McDowell, M. R. C., J. Wennell, P. A. Storr e J. Darzentas (1983) Gap acceptance and traffic conflict simulation as a measure of risk. 
Technical report, Transportation and Road Research Laboratory Supplementary Report 776.

Minderhoud, M. M. e P. H. L. Bovy (2001) Extended time-to-collision measures for road traffic safety assessment. Accident Analysis and Prevention, v. 33, p. 89-97. DOI: 10.1016/S0001-4575(00) 00019-1.

MOVIT, (2006) Braking distances. Disponível em: <http://www.movit. de/rahmen/stoptbl.htm>. (Acesso em 03/03/2007).

Neilsen, J. (2007) Stopping distances. Defensive Driving - Safe Driving Training SDT. Disponível em: <http://www.sdt.com.au>. (Acesso em 03/03/2007).

Oliveira, M. V. T. (2004) A Natureza dos Padrões de Variação EspaçoTemporal do Volume Veicular em Ambiente Urbano: Estudo de Caso em Fortaleza. Dissertação (Mestrado). Departamento de Engenharia de Transportes, Universidade Federal do Ceará. Fortaleza.

Perkins, S. e J. Harris (1968) Traffic conflict characteristics - accident potential at intersections. Highway Research Record, v. 225, p. 3543.

Saunier, N., N. Mourji e B. Agard (2011) Mining Microscopic Data of Vehicle Conflicts and Collisions to Investigate Collision Factors. Transportation Research Record: Journal of the Transportation Research Board, n. 2237, p. 41-50. DOI: 10.3141/2237-05.

Van der Horst, A. R. A. (1990) A Time-based Analysis of Road User Behaviour in Normal and Critical Encounters. Thesis (PhD), Delft University of Technology, 1990.

Xin, W., J. Hourdos, P. Michalopoulos e G. Davis (2008) The lessthanperfect driver: A model of collision-inclusive carfollowing behavior. In Proceeding of the $87^{\text {th }}$ Annual Meeting of the Transportation Research Board. DOI: 10.3141/2088-14. 